\title{
Global Solvability of the Maxwell-Bloch Equations from Nonlinear Optics
}

\author{
P. DONNAT \& J. RAUCH \\ Communicated by C. Dafermos
}

\section{§1. Introduction}

The nonlinear self-focusing of optical beams is a well-known and abundantly documented phenomenon [M,Sh]. The mechanism is simple. If the speed of propagation of electromagnetic waves decreases as a function of the intensity, then ray tracing suggests that a planar wavefront with intensity which is large at the center and decreases away from the center propagates so that the center lags behind the edges. This initial curvature translates into focusing after a finite period of time.

Interest in this phenomenon has been recently renewed with the advent of ultrashort and ultraintense pulses [Ro]. For ultraintense pulses, self-focusing is undesirable as it often leads to the extinction of the beam and to the destruction of very costly optical devices. In order to better understand focusing, with one goal being avoidance, good models are needed for the regions of space-time where laser beams focus.

The usual analysis of self-focusing uses the nonlinear Schrödinger (NLS) equation. This arises from more fundamental field equations by the slowly varying envelope approximation. One seeks an approximate solution in the form of a slowly varying field envelope times a rapidly oscillating term with linear phase function. A medium with an instantaneous cubic nonlinear response yields the NLS equation with cubic nonlinearity. This model predicts the instability of plane waves to transverse oscillations, and, thereby, the onset of self-focusing. It also lead to the prediction that it is possible for the self-focusing effect from the nonlinearity to exactly balance the diffusive effects from the Schrödinger equation leading to self-trapping [CGT]. It was soon observed both experimentally and theoretically that this trapping is not stable to perturbations in the two transverse dimensions. These early successes of the NLS established it and near relatives as the standard models for laser beam propagation.

In spite of this acceptance, this approximation remained little analyzed for many years. The slowly-varying-envelope approximation has recently been justified by [DR], taking advantage of many analytical advances in the work of JoLY, Metivier, RAUCH and Gues on nonlinear geometric optics. The approximation is 
the leading term in an asymptotic expansion valid as the wavelength tends to zero. In regions where the solution of the Schrödinger equation is regular, it gives a good approximation.

In the case of three-dimensional space, solutions of the NLS equation often develop singularities in finite time [Tal, K, G]. The standard interpretation is that this is symptomatic of catastrophic self-focusing. Our main result asserts that in the case of one fundamental model, the Maxwell-Bloch system, solutions remain regular for all $t \geqq 0$. They do not develop singularities in finite time. The singularity formation in the Schrödinger equation is symptomatic of the failure of the approximation. Though the approximate solution tends to infinity, the exact solution remains perfectly finite. The error in the approximation tends to infinity.

This is entirely analogous to the singularities in the amplitudes in linear geometric optics as one approaches caustics. Solutions of the linear Maxwell equations do not develop singularities, but, the asymptotic approximations do. To complete the picture for the nonlinear optics, one would need a more accurate asymptotic description near singularities of the Schrödinger equation. This appears to us to be a very difficult problem.

Near such singular points the behavior of the solution of the nonlinear Maxwell equations is not well understood. In fact, different fundamental field equations with the same NLS approximation may behave differently near the singular points. Thus an understanding of these behaviors can help in selecting correct models.

For extremely high fields, one expects that additional physical effects must be included, and that these should remove the singularity. Many attempts to modify the NLS model, none with much justification or success, have been suggested with this goal in mind. This includes adding to the NLS equation an ad hoc saturation of the nonlinear index [DM], multiphoton absorption [DLP], spatial and temporal nonparaxial terms [FF, Rot2], or group velocity dispersion [Rot1, MP, L]. Only for the saturated nonlinearity is there a rigorous proof that the singularity is eliminated.

In this paper, we study the more fundamental Maxwell equations. The medium is modelled as a gas of quantum mechanical systems with a finite number of energy levels. Such models, called Maxwell-Bloch (MB) systems, are often used to study the phenomena of resonance. We use it to model wave propagation. The electromagnetic field is modelled classically, while the medium is quantum mechanical. We prove rigorously that the solutions of this model remain regular provided that the initial condition is smooth.

The MB system produces a focusing or defocusing NLS equation as an asymptotic expansion, depending on the details of the finite state quantum system $[D, B C]$. For example, a three-level transition yields a focusing NLS equation under classical conditions [D]. Another example is provided by the low-lying energy levels for helium which show also that this medium is focusing in the low-frequency limit $[\mathrm{BL}]$.

For simplicity, we develop in this paper the simplest MB equation, that with two levels. The results remains valid for a finite number of levels independent of the focusing or defocusing behavior of approximating NLS. 
The unknowns in the MB system include three 3-vector fields; the electric and magnetic fields $E(t, x)$ and $B(t, x)$, and the polarization $P(t, x)$ of the medium. A scalar field $N(t, x)$ describes the difference between the number of electrons in the excited state and the ground state per unit volume. The number $N_{0}$ is the equilibrium value of $N$. In dimensionless notation the system is (see, for example, $[\mathrm{PP}])$

$$
\begin{aligned}
\partial_{t} B+\operatorname{curl} E & =0, \\
\partial_{t} E-\operatorname{curl} B & =-\partial_{t} P, \\
\partial_{t}^{2} P+\partial_{t} P / T_{2}+\Omega^{2} P & =c_{1} N E, \\
\partial_{t} N+\left(N-N_{0}\right) / T_{1} & =-c_{2}\left\langle\partial_{t} P, E\right\rangle .
\end{aligned}
$$

The nonnegative scalars $c_{1}$ and $c_{2}$ determine the strength of the coupling between the equations. The scalars $T_{1}$ and $T_{2}$ are called the inversion population lifetime and the homogeneous dephasing time. $\Omega$ is a resonant frequency which often corresponds to the principal optical spectral line of the medium.

Taking the divergence of (1.1) and (1.2) implies that

$$
\partial_{t} \operatorname{div}(E+P)=\partial_{t} \operatorname{div}(B)=0 .
$$

The physically relevant solutions are those which satisfy

$$
\operatorname{div}(E+P)=\operatorname{div}(B)=0 .
$$

Thanks to (1.5), this holds as soon as it holds at $t=0$, so (1.6) is only a linear constraint on the initial data.

Let

$$
U(t, x):=\left(E(t, x), B(t, x), P(t, x), \partial_{t} P(t, x), N(t, x)-N_{0}\right) .
$$

Equation (1.3) is written as a system for the pair $(P, Q)$ with $Q:=\partial_{t} P$,

$$
\partial_{i} P=Q, \quad \partial_{t} Q=-Q / T_{2}-\Omega^{2} P+c_{1} N E .
$$

The MB system then takes the form of a semilinear symmetric hyperbolic system for $U$ :

$$
\partial_{t} U=\sum_{1 \leqq j \leqq 3} A_{j} \partial_{j} U+F(U)
$$

where $\partial_{j}:=\partial / \partial x_{j}$, the $A_{j}$ are symmetric matrices, and $F: \mathbf{R}^{13} \rightarrow \mathbf{R}^{13}$ is a polynomial of degree two which vanishes at the origin. The standard local existence theorem for semilinear hyperbolic equations yields the following result.

Local Existence Theorem. If $s>\frac{3}{2}$ and $U(0,.) \in H^{s}\left(\mathbf{R}^{3}\right)$, then there is a $\left.\left.T_{*} \in\right] 0, \infty\right]$ and a unique $U \in C\left(\left[0, T_{*}\left[: H^{s}\left(\mathbf{R}^{3}\right)\right)\right.\right.$ which satisfies the Maxwell-Bloch system and attains these initial values. The solution depends continuously on the initial data in the sense that if $\varphi \in H^{s}\left(\mathbf{R}^{3}\right)$ and $T<T_{*}(\varphi)$, then there is an $H^{s}\left(\mathbf{R}^{3}\right)$ neighborhood $\mathcal{O}$ of $\varphi$ such that $T_{*}(\psi)>T$ for all $\psi \in \mathcal{O}$ and the map from initial data to solution is continuous from 10 to $C\left([0, T]: H^{s}\left(\mathbf{R}^{3}\right)\right)$. There is a $c>0$ such that $T_{*}>$ $c(s) /\left(1+\|U(0)\|_{H^{s}\left(\mathbf{R}^{3}\right)}\right)$. The values of $U$ at $(\underline{t}, \underline{x})$ depend only on the values of the initial data on the ball of radius $\underline{t}$ with center $\underline{x}$. 
Elements of the proof. For any $s \in \mathbf{R}$, the operator $\sum_{1 \leqq j \leqq 3} A_{j} \partial_{j} U$ is antiselfadjoint on $H^{s}\left(\mathbf{R}^{3}\right)$. Schauder's lemma shows that for $s>\frac{3}{2}$, the nonlinear term $F(U)$ is a locally Lipschitzian function on $H^{s}\left(\mathbf{R}^{3}\right)$. All the assertions of the lemma then follow by straightforward Picard iteration as in [Re, Thm. 1].

It follows that to prove global solvability for data $U(0,$.$) belonging to H^{s}$ for $s \geqq 2$ it suffices to prove the following $a$ priori estimate. For any $T \in] 0, \infty[$ and any $M>0$, there is a constant $C(T, M)$ such that if $\underline{t} \in] 0, T]$ and $U$ is a smooth compactly supported solution on $[0, t] \times \mathbf{R}^{3}$ such that

$$
\|U(0)\|_{H^{2}\left(\mathbf{R}^{3}\right)} \leqq M
$$

then

$$
\|U(t, .)\|_{H^{2}\left(\mathbf{R}^{3}\right)} \leqq C(T, M) \quad \text { for } 0 \leqq t \leqq \underline{t} .
$$

To prove that (1.9) is sufficient, one must show that if $U(0) \in H^{2}\left(\mathbf{R}^{3}\right)$ and $T>0$, then $T_{*}>T$. Choose $U_{n}(0) \in C_{0}^{\infty}\left(\mathbf{R}^{3}\right)$ converging to $U(0)$ in $H^{2}\left(\mathbf{R}^{3}\right)$. Let $M=\sup \left\|U_{n}(0)\right\|_{H^{2}\left(\mathbf{R}^{3}\right)}$ and $\delta=c(2) /(1+C(T, M))$ where $c(s)$ comes from the lower bound on the lifetime in the Local Existence Theorem. Then there are solutions $U_{n} \in C\left([0, \delta]: H^{2}\left(\mathbf{R}^{3}\right)\right)$. By uniqueness this is in $C\left([0, \delta]: H^{s}\left(\mathbf{R}^{3}\right)\right)$ for all $s \geqq 2$ and it follows that $U_{n}$ belongs to $C^{\infty}\left([0, \delta] \times \mathbf{R}^{3}\right)$. That the speed is finite implies that $U_{n} \in C_{(0)}^{\infty}\left([0, \delta] \times \mathbf{R}^{3}\right)$. Therefore (1.9) implies that the $C\left([0, \delta]: H^{2}\left(\mathbf{R}^{3}\right)\right)$ norm is at most $C(T, M)$. Restarting the process at $t=\delta$ produces $U_{n}$ in $C_{(0)}^{\infty}\left([0,2 \delta] \times \mathbf{R}^{3}\right)$ with the $C\left([0,2 \delta]: H^{2}\left(\mathbf{R}^{3}\right)\right)$ norm at most $C(T, M)$. Continuing this process yields $U_{n}$ in $C_{(0)}^{\infty}\left([0, T] \times \mathbf{R}^{3}\right)$ with the $C\left([0, T]: H^{2}\left(\mathbf{R}^{3}\right)\right)$ norm at most $C(T, M)$.

Choosing a weak star convergent subsequence yields a solution $U$ belonging to $L^{\infty}\left([0, T]: H^{2}\left(\mathbf{R}^{3}\right)\right)$. A simple $L^{2}$ uniqueness proof shows that $U$ is the solution and therefore belongs to $C\left([0, T]: H^{s}\left(\mathbf{R}^{3}\right)\right)$. Thus $T_{*}>T$.

In the next section we prove (1.9) for initial data which satisfy the divergence condition (1.6).

Main Theorem. If $s \geqq 2$ and the initial data $U(0,.) \in H^{s}\left(\mathbf{R}^{3}\right)$ satisfy (1.6), then $T_{*}=\infty$. That is, there is a tnique global solution belonging to $C\left(\left[0, \infty\left[: H^{s}\left(\mathbf{R}^{3}\right)\right)\right.\right.$.

The proof proceeds by a sequence of a priori estimates finally leading to the construction of the function $C(T, M)$. The estimates derived in $\$ 2$ through $\$ 5$ do not use the divergence equation (1.6), and depend only on $T$ and

$$
m:=\|U(0)\|_{H^{1}\left(\mathbf{R}^{3}\right)}+\left\|P(0), \partial_{t} P(0), N(0)\right\|_{L^{\infty}\left(\mathbf{R}^{3}\right)} .
$$

\section{§2. The Fundamental $L^{2}$ Estimate}

The law of energy balance is demonstrated by taking the scalar product of (1.1) with $N_{0} B,(1.2)$ with $N_{0} E$, and (1.3) and $\partial_{t} P / c_{1}$. Equation (1.4) is multiplied by $\left(N-N_{0}\right) / c_{2}$ and the resulting expressions are added. The products of the 
right-hand sides with the multipliers is

$$
-\partial_{t} P \cdot\left|N_{0}\right| E-c_{1} N E \cdot \partial_{t} P / c_{1}+c_{2} \partial_{t} P \cdot E\left(N-N_{0}\right) / c_{2}=0 .
$$

This cancellation and a similar one presented in the next step are the keys to the existence proof. Using the vector identity $B \cdot \operatorname{curl} E-E \cdot \operatorname{curl} B=\operatorname{div}(E \wedge B)$ yields the dissipation law

$$
\partial_{t} e+\operatorname{div}\left(N_{0} E \wedge B\right)=-\frac{\left|\partial_{t} P\right|^{2}}{T_{2} c_{1}}-\frac{\left(N-N_{0}\right)^{2}}{T_{1} c_{2}}
$$

where

$$
2 e(t, x):=N_{0}|E|^{2}+N_{0}|B|^{2}+\frac{\left|\partial_{t} P\right|^{2}+\Omega^{2} P^{2}}{c_{1}}+\frac{\left(N-N_{0}\right)^{2}}{c_{2}} .
$$

The consequences of (2.1) are our only estimates which do not grow in time.

Integrating the dissipation law over the truncated cone $|x| \leqq R-t$, $0<t<T<R$ shows that the MB system has speed of propagation at most 1 . Letting $R$ tend to infinity shows that solutions $U$ which are suitably small at infinity satisfy the energy dissipation identity

$$
\int_{\mathbf{R}^{3}} e(t, x) d x+\int_{0}^{t} \int_{\mathbf{R}^{3}} \frac{\left|\partial_{t} P\right|^{2}}{T_{2} c_{1}}+\frac{\left(N-N_{0}\right)^{2}}{\left(T_{1} c_{2}\right)} d x d t=\int_{\mathbf{R}^{3}} e(0, x) d x
$$

In particular, $\int_{\mathbf{R}^{3}} e(t, x) d x$ is a decreasing function of time.

\section{§. An $L^{\infty}$ estimate for $P, \partial_{t} P$, and $N$}

The key to proving global solvability and global regularity is to show that solutions are bounded on $[0, T] \times \mathbf{R}^{3}$. In this section we prove such boundedness for $P, Q:=\partial_{t} P$, and $N$.

Take the scalar product of (1.3) with $2 \partial_{1} P / c_{1}$ and multiply (1.4) by $2 N / c_{2}$. Add the resulting expressions. The cubic terms from the right-hand sides cancel exactly. This leaves

$$
\partial_{t}\left(\frac{\left|\partial_{t} P\right|^{2}+\Omega^{2}|P|^{2}}{c_{1}}+\frac{N^{2}}{c_{2}}\right)+\frac{2\left|\partial_{t} P\right|^{2}}{c_{1} T_{2}}+\frac{2 N^{2}}{c_{2} T_{1}}=\frac{2 N N_{0}}{c_{2} T_{1}}
$$

The right-hand side is less than or equal to

$$
\frac{1}{c_{2} T_{1}}\left(2 N^{2}+N_{0}^{2} / 2\right)
$$

The first term is absorbed by last term on the left-hand side of (3.1). The second term is independent of time. Integrating the differential inequality shows that for all $t, x$ with $t \geqq 0$,

$$
\begin{aligned}
\frac{\left|\partial_{t} P(t, x)\right|^{2}+\Omega^{2}|P(t, x)|^{2}}{c_{1}} & +\frac{N(t, x)^{2}}{c_{2}} \\
& \leqq \frac{\left|\partial_{t} P(0, x)\right|^{2}+\Omega^{2}|P(0, x)|^{2}}{c_{1}}+\frac{N(0, x)^{2}}{c_{2}}+\frac{t N_{0}^{2}}{2 c_{2} T_{1}}
\end{aligned}
$$


This gives a pointwise bound on $P, \partial_{t} P$ and $N$ which grows at most like $\sqrt{t}$. It is worth noting that one could get pointwise bounds for negative time which grow like $\exp (c \sqrt{|t|})$.

\section{§4. $H^{1}$ Estimates}

The $L^{\infty}$ bounds for $N$ and $\partial_{t} P$ control one of the factors in each of the quadratic nonlinear terms in the MB system. This together with the same cancellations which gave us the $L^{2}$ bounds of $\$ 2$ suffice to derive $H^{1}$ bounds.

Denote by $\partial_{\mu}$ partial differentiation with respect to $t$ or $x_{j}, 1 \leqq j \leqq 3$, depending on whether $\mu=0$ or $1 \leqq \mu \leqq 3$. Apply $\partial_{\mu}$ to each of the equations (1.1)-(1.4). Then multiply the differentiated equation by $\partial_{\mu}$ applied to the corresponding multiplier from $\$ 2$. Add the resulting expressions. The cubic terms in the sum are equal to

$$
\partial_{\mu}(N E) . \partial_{\mu} \partial_{t} P-\left(\partial_{\mu} N\right) \partial_{\mu}\left(\partial_{t} P . E\right) .
$$

The key is that the two terms in this difference with $E$ undifferentiated cancel. The other terms have a factor of $N$ or $\partial_{t} P$ for which we have an $L^{\infty}$ estimate. For $t \geqq 0$ let

Let

$$
Y(t):=\sup _{x \in \mathbf{R}^{3}}(\text { right-hand side of }(3.2))^{1 / 2} .
$$

$$
2 e_{1}(t, x):=\sum_{\mu=0}^{3} N_{0}\left|\partial_{\mu} E\right|^{2}+N_{0}\left|\partial_{\mu} B\right|^{2}+\frac{\left|\partial_{\mu} \partial_{t} P\right|^{2}+\Omega^{2}\left|\partial_{\mu} P\right|^{2}}{c_{1}}+\frac{\left|\partial_{\mu}\left(N-N_{0}\right)\right|^{2}}{c_{2}} .
$$

The above computation shows that

$$
\left|\partial_{t} \int_{\mathbf{R}^{3}} e_{1}(t, x) d x\right| \leqq c Y(t) \int_{\mathbf{R}^{3}} e_{1}(t, x) d x .
$$

Let

$$
Y_{1}(t):=\int_{\mathbf{R}^{3}} e_{1}(t, x) d x
$$

Then (4.3) implies that for $t \geqq 0$,

$$
Y_{1}(t) \leqq Z(t)
$$

where $Z(t)$, defined and smooth on $[0, \infty[$, is the solution of

$$
\frac{d Z(t)}{d t}=c Y(t) Z(t), \quad Z(0)=Y_{1}(0)
$$

\section{\$5. $C^{\alpha}$ bound for the divergence-free part of $E$}

The Fourier transform of the divergence and curl of $E$ are equal to $\langle\xi, \widehat{E}\rangle$ and $\xi \wedge \hat{E}$ respectively. Thus, decomposing $\hat{E}(\xi)$ into the part parallel and orthogonal 
to $\xi$ is the $L^{2}\left(\mathbf{R}^{3}\right)$ orthogonal projection on the irrotational and divergence-free parts.

Define a smooth projection-valued function on $\mathbf{R}_{\xi}^{3} \backslash 0$ by

$$
\Pi(\xi) v=v-\xi\langle\xi, v\rangle\langle\langle\xi, \xi\rangle .
$$

Then $\Pi(D)$ defined by

$$
\Pi(D) E:=\mathscr{F}^{-1}(\Pi(\xi)(\mathscr{F} E)(\xi))
$$

is a convolution operator which is a classical singular integral operator of degree zero. $\Pi(D)$ is the $L^{2}$ orthogonal projection onto the divergence-free vector fields along the irrotational fields.

For any vector $v$ and any $\xi$,

$$
\xi \wedge(\xi \wedge v)=-|\xi|^{2} \Pi(\xi) v .
$$

It follows that

$$
\text { curl curl } \Pi(D)=\Pi(D) \Delta, \quad \text { curl } \operatorname{curl}(I-\Pi(D))=0 .
$$

Taking the time derivative of (1.2) and then using (1.1) to eliminate $B$ yields

$$
E_{t t}-\operatorname{curl} \operatorname{curl} E=-P_{t t} \text {. }
$$

Applying $\Pi(D)$ and using (1.3) yields

$$
\left(\partial_{t t}-\Delta\right) \Pi(D) E=-\Pi(D)\left(c_{1} N E-\Omega^{2} P-\partial_{t} P / T_{2}\right) .
$$

The estimates of $\S 4$ imply that the $P$ and $\partial_{t} P$ terms on the right are bounded in the space of continuous functions with values in $H^{1}$. The next result shows that we can bound $N(t) E(t)$ in $H^{s}\left(\mathbf{R}^{3}\right)$ for any $s<\frac{2}{3}$.

Lemma. For any $s<\frac{2}{3}$, pointwise multiplication of functions defines a continuous bilinear map of $\left(L^{\infty} \cap H^{1}\right)\left(\mathbf{R}^{3}\right) \times H^{1}\left(\mathbf{R}^{3}\right)$ to $H^{s}\left(\mathbf{R}^{3}\right)$.

Proof. The proof is by two interpolations. Sobolev's Lemma implies that if $\rho>\frac{3}{2}$, then multiplication is continuous from $H^{\rho}\left(\mathbf{R}^{3}\right) \times H^{0}\left(\mathbf{R}^{3}\right)$ to $H^{0}\left(\mathbf{R}^{3}\right)$. Schauder's Lemma implies continuity from $H^{\rho}\left(\mathbf{R}^{3}\right) \times H^{\rho}\left(\mathbf{R}^{3}\right)$ to $H^{\rho}\left(\mathbf{R}^{3}\right)$. Interpolation proves continuity from $H^{\rho}\left(\mathbf{R}^{3}\right) \times H^{\sigma}\left(\mathbf{R}^{3}\right)$ to $H^{\sigma}\left(\mathbf{R}^{3}\right)$ for all $0 \leqq \sigma \leqq \rho$.

Let $\mathbf{X}:=\left(L^{\infty} \cap H^{1}\right)\left(\mathbf{R}^{3}\right)$. Taking $\sigma=1$ shows that multiplication is continuous from $\mathbf{X} \times H^{\rho}$ to $H^{1}$.

Since $\mathbf{X} \subset L^{\infty}$, it follows that multiplication is continuous from $\mathbf{X} \times H^{0}\left(\mathbf{R}^{3}\right)$ to $H^{0}\left(\mathbf{R}^{3}\right)$.

Interpolating between these two results with interpolation parameter $\theta=1 / \rho$ shows that multiplication is continuous from $\mathbf{X} \times H^{1}\left(\mathbf{R}^{3}\right)$ to $H^{1 / \rho}\left(\mathbf{R}^{3}\right)$. Since this is valid for all $\rho>\frac{3}{2}$, the proof is complete.

Given the $L^{\infty} \cap H^{1}$ bounds for $N$ and the $H^{1}$ bounds for $E$, it follows that $E N$ and therefore $\Pi(D)(E N)$ are bounded in the space of continuous functions of $t$ with values in $H^{s}\left(\mathbf{R}^{3}\right)$ for any $s<\frac{2}{3}$. 
Applying standard estimates for d'Alembert's wave equation (see, for example, [Ra, \$3.7]) to $(5.5)$ bounds $\Pi(D) E$ as a continuous function with values in $H^{s^{+1}}\left(\mathbf{R}^{3}\right)$ and $\partial_{t} \Pi(D) E$ as a continuous function with values in $H^{s}\left(\mathbf{R}^{3}\right)$.

Sobolev's lemma shows that $H^{s+1}\left(\mathbf{R}^{3}\right) \subset C^{s-1 / 2}\left(\mathbf{R}^{3}\right)$ for $\frac{1}{2}<s<1$. The limiting value $s=\frac{2}{3}$ yields $C^{1 / 6}$. Thus for $0<\alpha<\frac{1}{6}$, one has a bound

$$
\|\Pi(D) E(t)\|_{C^{\alpha}\left(\mathbf{R}^{3}\right)} \leqq C(T, m, \alpha) \quad \text { for } 0 \leqq t \leqq t \leqq T,
$$

where $m$ is defined in (1.10).

\section{§6. $L^{\infty}$ estimate for the irrotational part of $E$}

To bound the sup norm of the irrotational part $(I-\Pi(D)) E$ of $E$, we use the divergence equation (1.6) and second derivatives of $U$. The second derivatives have not yet been estimated. A Gronwall argument in the next section completes the demonstration.

Equation (1.6) implies that

$$
(I-\Pi(D)) E=-(I-\Pi(D)) P .
$$

The right-hand side is an operator of order zero applied to the bounded function $P$. Unfortunately such operators do not map bounded functions to bounded functions. An observation, dating at least to YuDovich [Y], is that a logarithmic correction is a partial remedy. Applying the version given in Proposition B.1.A of [T] yields

$$
\|(I-\Pi(D)) E(t)\|_{L^{\infty}\left(\mathbf{R}^{3}\right)} \leqq c\|P(t)\|_{L^{\infty}\left(\mathbf{R}^{3}\right)} \log \left(2+\|P(t)\|_{H^{2}\left(\mathbf{R}^{3}\right)}\right) .
$$

Combining this with the estimate of the previous section one has for any solution on $[0, t]$ and $t \leqq \underline{t}$,

$$
\left.\|E(t)\|_{L^{\infty}\left(\mathbf{R}^{3}\right)} \leqq c(m, T)+\text { (right-hand side of } 6.2\right) .
$$

\section{§. The final $H^{2}$ estimate}

In this section the second derivatives of a solution on $[0, t]$ with $t \leqq T$ are estimated by an energy argument related to that used for the $L^{2}$ and $H^{i}$ estimates. A subtlety is that the differential inequality which we derive is logarithmically superlinear because of (6.2) and (6.3).

For each $\mu, v$, apply $\partial_{\mu} \partial_{\nu}$ to each of the equations (1.1)-(1.4). Multiply the resulting equations by $\partial_{j} \partial_{w}$ applied to the corresponding multiplier from $\$ 2$. Add the resulting expressions. The contribution of the right-hand sides is equal to

$$
-\left(\partial_{\mu} \partial_{\nu} \partial_{t} P\right) . \partial_{\mu} \partial_{\nu} N_{0} E+\left(\partial_{\mu} \partial_{\nu}(N E)\right) \cdot \partial_{\mu} \partial_{\nu} \partial_{t} P-\partial_{\mu} \partial_{v}\left\langle\partial_{t} P, E\right\rangle . \partial_{\mu} \partial_{\nu}\left(N-N_{0}\right)
$$

There are four terms. The two quadratic terms cancel. 
Let

$$
\begin{aligned}
2 e_{\mu v}(t, x):= & N_{0}\left|\partial_{\mu} \partial_{\nu} E(t, x)\right|^{2}+N_{0}\left|\partial_{\mu} \partial_{\nu} B(t, x)\right|^{2} \\
& +\frac{\left|\partial_{\mu} \partial_{v} \partial_{t} P(t, x)\right|^{2}+\Omega^{2}\left|\partial_{\mu} \partial_{\nu} P(t, x)\right|^{2}}{c_{1}}+\frac{\left|\partial_{\mu} \partial_{v}\left(N(t, x)-N_{0}\right)\right|^{2}}{c_{2}} .
\end{aligned}
$$

The above computation yields a balance law for second derivatives:

$$
\begin{aligned}
\partial_{t}\left(\partial_{\mu} \partial_{v} e\right)+\operatorname{div}\left(N_{0} \partial_{\mu} \partial_{v} E\right. & \left.\wedge \partial_{\mu} \partial_{v} B\right)+\frac{\left|\partial_{\mu} \partial_{v} \partial_{t} P\right|^{2}}{T_{2} c_{1}}+\frac{\left(\partial_{\mu} \partial_{v} N\right)^{2}}{T_{1} c_{2}} \\
& =\left(\partial_{\mu} \partial_{v}(N E)\right) . \partial_{\mu} \partial_{v} \partial_{t} P-\left(\partial_{\mu} \partial_{v} N\right) \partial_{\mu} \partial_{v}\left(\partial_{t} P . E\right)
\end{aligned}
$$

Let

$$
Y_{2}(t):=\int_{\mathbf{R}^{3}} e(t, x)+e_{1}(t, x)+\sum_{\mu, v} e_{\mu \nu}(t, x) d x .
$$

Adding (2.3), (4.4), and the result of integrating (7.2) over $\mathbf{R}^{3}$ yields

$$
Y_{2}^{\prime}(t) \leqq c\|U(t)\|_{H^{2}\left(\mathbf{R}^{3}\right)}^{2}+\sum_{\mu, v} \int_{\mathbf{R}^{3}}\left(\partial_{\mu} \partial_{v}(N E)\right) . \partial_{\mu} \partial_{v} \partial_{t} P-\left(\partial_{\mu} \partial_{v} N\right) \partial_{\mu} \partial_{v}\left(\partial_{t} P . E\right) d x
$$

To estimate the integral on the right in (7.4), note that from the derivatives of the products there are two types of terms, those for which there is a factor which is not differentiated and those for which two factors are differentiated, one time each. Terms of the first type are dominated by

$$
c\left\|\left(E, N, \partial_{t} P\right)(t)\right\|_{L^{\infty}\left(\mathbf{R}^{3}\right)}\|U(t)\|_{H^{2}\left(\mathbf{R}^{3}\right)}^{2} .
$$

Terms of the second type are dominated by

$$
c\left\|\partial\left(E, N, \partial_{t} P\right)(t)\right\|_{L^{4}\left(\mathbf{R}^{3}\right)}^{2}\|U(t)\|_{H^{2}\left(\mathbf{R}^{3}\right)}
$$

The interpolation inequality

$$
\left\|\partial\left(E, N, \partial_{t} P\right)(t)\right\|_{L^{4}\left(\mathbf{R}^{3}\right)}^{2} \leqq c\left\|\left(E, N, \partial_{t} P\right)(t)\right\|_{L^{\infty}\left(\mathbf{R}^{3}\right)}\left\|\left(E, N, \partial_{t} P\right)(t)\right\|_{H^{2}\left(\mathbf{R}^{3}\right)}
$$

shows that (7.6) is also dominated by (7.5).

Next, the estimates (3.2) and (6.3) imply that

$$
\left\|\left(E, N, \partial_{t} P\right)(t)\right\|_{L^{\infty}\left(\mathbf{R}^{3}\right)} \leqq c(m, T) \log \left(2+\|U(t)\|_{H^{2}\left(\mathbf{R}^{3}\right)}\right) .
$$

Using these estimates in (7.4) yields a differential inequality for $0 \leqq t \leqq t \leqq T$ :

$$
Y_{2}^{\prime}(t) \leqq c(m, T)\|U(t)\|_{H^{2}\left(\mathbf{R}^{3}\right)}^{2} \log \left(2+\|U(t)\|_{H^{2}\left(\mathbf{R}^{3}\right)}\right) .
$$

Finally, note that $Y_{2}$ is larger than a constant times the square of the $H^{2}$ norm of $U(t)$, so one has

$$
Y_{2}^{\prime}(t) \leqq c(m, T) Y_{2}(t) \log \left(2+Y_{2}(t)\right) .
$$

Thus for $0 \leqq t \leqq t \leqq T$,

$$
Y_{2}(t) \leqq Z_{2}(t)
$$


where $Z_{2}(t)$ is the solution of the globally solvable initial-value problem

$$
Z_{2}^{\prime}(t)=c(m, T) \log \left(2+Z_{2}(t)\right) Z_{2}(t) \quad Z_{2}(0)=Y_{2}(0) .
$$

This completes the proof of the estimate 1.9 and therefore the proof of the Main Theorem.

The bound for the second derivative grows very rapidly with time, in fact, faster than a multiple exponential. We do not know whether the solutions actually grow anywhere nearly this fast.

\section{§8. Global $H^{1}$ solutions}

By using the fact that the estimates of $\$ 2$ and $\$ 3$ depend only on $m$ defined in (1.10), it is not hard to prove the following result about global weak solutions.

Theorem. For arbitrary $U(0) \in H^{1}\left(\mathbf{R}^{3}\right)$ such that $\left(N(0), P(0), \partial_{i} P(0)\right) \in L^{\infty}\left(\mathbf{R}^{3}\right)$, there is a unique solution such that for all $T>0, U \in L^{\infty}\left([0, T]: H^{1}\left(\mathbf{R}^{3}\right)\right)$ and $\left(N, P, \partial_{t} P\right) \in L_{.}^{\infty}\left([0, T] \times \mathbf{R}^{3}\right)$. The solution satisfies the energy dissipation identity (2.3) and has speed of propagation at most unity.

Remarks. 1. If $U(0)$ is in $L^{\infty}$, the estimates in $\S 5$ suffice to show that $\Pi(D) E$ is bounded on $[0, T] \times \mathbf{R}^{3}$. A similar argument shows that $B$ is bounded. The only part of $U$ for which sup norm bounds are not known is the irrotational part $(I-\Pi(D)) E$ of $E$.

2. Since there is an $L^{2}$ dissipation law with a natural physical interpretation, and since the nonlinearities are quadratic, it would be natural to seek weak solutions with $U(0)$ square integrable. This remains an open problem.

Proof of Theorem. The solutions are constructed as limits of approximate solutions $U_{\varepsilon}$ whose initial data are equal to $J_{\varepsilon} U(0)$ where $J_{\varepsilon}$ is a standard spatial mollifier:

$$
J_{\varepsilon}(\varphi):=\int_{\mathbf{R}^{3}} j(y) \varphi(x-\varepsilon y) d y, \quad \text { where } j \in C_{0}^{\infty}\left(\mathbf{R}^{3}\right), \quad \int_{\mathbf{R}^{3}} j(y) d y=1 .
$$

The estimates of $\$ \S 2-4$ show that the approximate solutions are bounded in the space of continuous functions with values in $H^{1}$ and that $\left(N_{\varepsilon}, P_{\varepsilon}, \partial_{t} P_{\varepsilon}\right)$ is bounded in $L^{\infty}\left([0, T] \times \mathbf{R}^{3}\right)$.

Since each of the quadratic nonlinear terms has one bounded factor, the differential equations $(1.1)-(1.4)$ show that the family $\partial_{t} U_{\varepsilon}$ is bounded in the space of continuous functions of time with values in $L^{2}\left(\mathbf{R}^{3}\right)$.

Combining the finite domain of dependence and the local compactness of $H^{1}$ in $L^{2}$ we can apply the Arzelà-Ascoli theorem to choose a subsequence strongly convergent in $C\left(\left[0, \infty\left[: L^{2}\left(\mathbf{R}^{3}\right)\right)\right.\right.$ to a limit $U$.

The strong convergence shows that we can pass to the limit in the nonlinear terms of the MB system since they are quadratic. Similarly one can pass to the limit in the energy dissipation law. Thus $U$ satisfies the system and the law. 
The $L^{2}$ convergence and $L^{\infty}$ boundedness imply that $\left(N_{\varepsilon}, P_{\varepsilon}, \partial_{t} P_{\varepsilon}\right)$ converges weak star in $L^{\infty}\left([0, T] \times \mathbf{R}^{3}\right)$. Similarly, the solution constructed is in $L^{\infty}\left([0, T]: H^{1}\left(\mathbf{R}^{3}\right)\right)$. Thus

$$
U \in L^{\infty}\left([0, T] \times \mathbf{R}^{3}\right) \cap L^{\infty}\left([0, T]: H^{1}\left(\mathbf{R}^{3}\right)\right) .
$$

This completes the existence proof.

The proof of uniqueness depends on the cancellations leading to the $L^{2}$ estimate in $\S 2$. Suppose that one had two solutions, indicated by the superscripts 1 and 2. Subtract the equations (1.1)-(1.4) for the two solutions. Multiply the equations by $N_{0}\left(B^{1}-B^{2}\right), N_{0}\left(E^{1}-E^{2}\right),\left(\partial_{t} P^{1}-\partial_{t} P^{2}\right) / c_{1}$, and $\left(N^{1}-N^{2}\right) / c_{2}$. The contribution of the right-hand sides is

$$
\begin{aligned}
- & \left(\partial_{t} P^{1}-\partial_{t} P^{2}\right) \cdot N_{0}\left(E^{1}-E^{2}\right) \\
& +\left(N^{1} E^{1}-N^{2} E^{2}\right) \cdot\left(\partial_{t} P^{1}-\partial_{t} P^{2}\right)-\left(\partial_{t} P^{1} \cdot E^{1}-\partial_{t} P^{2} \cdot E^{2}\right)\left(N^{1}-N^{2}\right) .
\end{aligned}
$$

The first product is quadratic. Of the eight cubic terms, the two terms which only have superscripts 1 cancel. Similarly the two terms with only superscripts 2 cancel.

Let

$$
\begin{aligned}
2 f(t, x):= & N_{0}\left|E^{1}-E^{2}\right|^{2}+N_{0}\left|B^{1}-B^{2}\right|^{2} \\
& +\frac{\left|\partial_{t}\left(P^{1}-P^{2}\right)\right|^{2}+\Omega^{2}\left|P^{1}-P^{2}\right|^{2}}{c_{1}}+\frac{\left(N^{1}-N^{2}\right)^{2}}{c_{2}} .
\end{aligned}
$$

Then the quadratic terms in (8.1), are bounded by $c f(t, x)$. The above cancellation leaves four cross terms. Using the divergence identity from $\$ 2$, one finds a balance law

$$
\begin{aligned}
\partial_{t} f(t, x) & +q(t, x)+\operatorname{div}\left(N_{0}\left(E^{1}-E^{2}\right) \wedge\left(B^{1}-B^{2}\right)\right) \\
& \leqq c f(t, x)-\partial_{t} P^{1} N^{2} \cdot E^{2}-\partial_{t} P^{2} N^{1} \cdot E^{1}+N^{1} \partial_{t} P^{2} \cdot E^{2}+N^{2} \partial_{t} P^{1} \cdot E^{1},
\end{aligned}
$$

where

$$
q(t, x):=\frac{\left|\partial_{t} P^{1}-\partial_{t} P^{2}\right|^{2}}{T_{2} c_{1}}+\frac{\left(N^{1}-N^{2}\right)^{2}}{T_{1} c_{2}} \geqq 0 .
$$

The last four terms in (8.3) simplify to

$$
\left(E^{1}-E^{2}\right) \cdot\left(\partial_{t} P^{2} N^{1}-\partial_{t} P^{1} N^{2}\right)=\left(E^{1}-E^{2}\right) \cdot\left(\partial_{t} P^{2}\left(N^{1}-N^{2}\right)+N^{2}\left(\partial_{t} P^{2}-\partial_{t} P^{1}\right)\right) \text {. }
$$

Since $\partial_{t} P^{2}$ and $N^{2}$ are bounded on $[0, T] \times \mathbf{R}^{3}$, one finds that for $0 \leqq t \leqq T$,

$$
\partial_{i} f(t, x)+\operatorname{div}\left(N_{0}\left(E^{1}-E^{2}\right) \wedge\left(B^{1}-B^{2}\right)\right) \leqq c f(t, x) .
$$

As in the existence part, the differential equation shows that $\partial_{t}\left(U^{1}-U^{2}\right)$ is bounded with values in $L^{2}\left(\mathbf{R}^{3}\right)$. Let $Y_{3}(t):=\int_{\mathbf{R}^{3}} f(t, x) d x$. Integrate (8.5) over $\left([0, t] \times \mathbf{R}^{3}\right)$. Integration by parts is justified since $U^{1}-U^{2}$ belongs to $H^{1}\left([0, t] \times \mathbf{R}^{3}\right)$. Since $U^{1}(0)=U^{2}(0)$, it follows that $Y_{3}(0)=0$ and the above 
integration yields

$$
Y_{3}(t) \leqq c \int_{0}^{t} Y_{3}(s) d s .
$$

Gronwall's inequality implies that $Y_{3}(t)=0$ for all $t \geqq 0$ proving uniqueness.

Acknowledgements. This work was begun while RAUCH was visiting the Centre de Mathématiques Pures et Appliquées at the Ecole Polytechnique, Palaiseau. He thanks this center and in particular J-M. BONY, J-P. BOURGUINON, J-C. NEDELEC and P-A. RAviART for their hospitality. We have greatly benefitted from our participation in the CNRS Groupe de Recherche (GdR) d'Optique Non Linéaire organized by P. GLorIEux. In particular, the frequent advice and encouragement of C. GouÉdARD, J-L. JOLY, G. METIVIER and A. Migus were crucial in forming our ideas. It was Professor RAVIART who suggested that we work together on these problems, and we thank him most heartily. The work of RAUCH was partially supported by the Office of Naval Research and the National Science Foundation under grants N O14 $92 \mathrm{~J} 1245$ and DMS 9003256.

\section{References}

[BL] D. M. BISHOP \& B. LAM, Ab initio study of third order nonlinear optical properties of helium, Phys. Rev. A, 37, 464-469 (1988).

[BC] P. Butcher \& D. CotTER, The Elements of Nonlinear Optics, Cambridge Univ. Press, Cambridge, UK, 1990.

[CGT] R. Y. ChIAO, E. GARMIRE \& C. H. TOWnEs, Self-trapping of optical beams, Phys. Rev. Letters, 13, 479-480 (1964).

[DM] E. L. DAwes \& J. H. MARBURGER, Computer studies in self-focusing, Phys. Rev., $179,862-867(1969)$.

[DLP] A. P. Dyshko, V. N. Lugovor \& A. M. Prokhorov, Development of an optical waveguide in the propagation of light in nonlinear medium Sov. Phys. JETP, 34, 1235-1241 (1972).

[D] P. DONNAT, Quelque contributions mathématiques en optique nonlinéaire, Thèse, Ecole Polytechnique, Paris, 1994.

[DR] P. DONNAT \& J. RAUCH, Dispersive nonlinear geometric optics, J. Math. Phys. to appear.

[FF] M. D. FEIT \& J. A. FLECK, Beam non paraxiality, filament formation and beam break up in the self-focusing of optical beam, J. Opt. Soc. Am. B, 5, 633-640 (1988).

[G] R. T. GLASSEX, On the blow-up of solutions of the Cauchy problem for the nonlinear Schrödinger equation, J. Math. Physics, 18, 1794-1797 (1977).

[JMR] J.-L. JOLY, G. METrVIER \& J. RAUCH, Global solvability of the anharmonic oscillator model from nonlinear optics, SI AM J. Math. Anal., 27, 905-913 (1996).

[H] L. HörmANDER, Non-linear Hyperbolic Differential Equations, Lecture Notes, Lund University, 1987.

[K] P. L. KELLEY, Self-focussing of optical beams, Phys. Rev. Letters, 15, 1005-1008 (1965).

[L] G. G. Luther, A. C. Newell \& J. V. MAloney, Normal dispersion arrests critical collapse, preprint.

[MP] V. MALKIN \& G. PAPANICOLAOU, On self-focusing of short laser pulses, preprint (1993). 
[M] J. H. Marburger, Self-focussing theory, Prog. Quant. Elect. 4, 35-110 (1975).

[NM] A. Newell \& J. Moloney, Nonlinear Optics, Addison-Wesley, Reading, Mass., 1992.

[PP] R. PANTEll \& H. PuthofF, Fundamentals of Quantum Electronics, Wiley, New York, 1969.

[Ra] J. RAUCH, Partial Differential Equations, Springer-Verlag, New York, 1991.

[Re] M. ReED, Abstract Non-Linear Wave Equations, Springer-Verlag, New York, 1975.

[Rot1] J. E. ROTHENBERG, Pulse splitting during self-focussing in normally dispersive media. Opt. Letters, 17, 583-585 (1992).

[Rot2] J. E. ROTHENBERG, Breakdown of the slowly varying envelope approximation in the self-focussing of femtosecond pulses. Opt. Letters, 17, 1340-1342 (1992).

[Ro] C. Rouyer, E. Mazataud, I. Allais, A. Pierre, S. Seznec, C. Sauteret, G. Mourou \& A. Migus: Generation of 50TW femtosecond pulses in a Ti:sapphire/Nd: glass chain, Opt. Letters, 18, 214-216 (1993).

[Sh] Y. R. SHEN, The Principles of Nonlinear Optics, Wiley-Interscience, New York, 1984.

[T] M. TAYLoR, Pseudodifferential Operators and Nonlinear Optics, Birkhäuser, Boston, 1991.

[Tal] V. I. TALANOv, Self-focusing of wave beams in nonlinear media, J. Exper. Theor. Phys. Lett., 2, 138-141 (1965).

[Y] V. YUdovich, Nonstationary flows of an ideal incompressible fluid, Zh. Vych. Math., 3, 1032-1066 (1963).

Commisariat à l'Energie Atomique

Centre d'Etude de Limeil Valenton

94195 Villeneuve St. Georges cedex, France

and

Department of Mathematics

University of Michigan

Ann Arbor, Michigan 48109

(Accepted June 22, 1995) 